\title{
Perturbation formula for the two-phase membrane problem
}

\author{
Farid Bozorgnia
}

Correspondence: bozorg@math.ist. utl.pt

Faculty of Sciences, Persian Gulf University, Bushehr 75168, Iran

\section{Abstract}

A perturbation formula for the two-phase membrane problem is considered. We perturb the data in the right-hand side of the two-phase equation. The stability of the solution and the free boundary with respect to perturbation in the coefficients and boundary value is shown. Furthermore, continuity and differentiability of the solution with respect to the coefficients are proved.

Keywords: Free boundary problems, Two-phase membrane, Perturbation

\section{Introduction}

Let $\lambda^{ \pm}: \Omega \rightarrow \mathbb{R}$ be non-negative Lipschitz continuous functions, where $\Omega$ is a bounded open subset of $\mathbb{R}^{n}$ with smooth boundary. Assume further that $g \in W^{1,2}(\Omega) \cap L^{\infty}(\Omega)$ and $g$ changes sign on $\partial \Omega$. Let $K=\left\{v \in W^{1,2}(\Omega): v-g \in W_{0}^{1,2}(\Omega)\right\}$. Consider the functional

$$
I(v)=\int_{\Omega}\left(\frac{1}{2}|\nabla v|^{2}+\lambda^{+} \max (v, 0)-\lambda^{-} \min (v, 0)\right) d x,
$$

which is convex, weakly lower semi-continuous and hence attains its infimum at some point $u \in K$. The Euler-Lagrange equation corresponding to the minimizer $u$ is given by Weiss [1] and is called the two-phase membrane problem:

$$
\begin{cases}\Delta u=\lambda^{+} \chi_{\{u>0\}}-\lambda^{-} \chi_{\{u>0\}} & \text { in } \Omega, \\ u=g & \text { on } \partial \Omega,\end{cases}
$$

where $\chi_{A}$ denotes the characteristic function of the set $A$, and

$$
\Gamma(u)=\partial\{x \in \Omega: u(x)>0\} \cup \partial\{x \in \Omega: u(x)<0\} \cap \Omega
$$

is called the free boundary. The free boundary consists of two parts:

$$
\Gamma^{\prime}(u)=\Gamma(u) \cap\{x \in \Omega: \nabla u(x)=0\}
$$

and

$$
\Gamma^{\prime \prime}(u)=\Gamma(u) \cap\{\nabla u(x) \neq 0\} .
$$

By $\Omega^{+}(u)$ and $\Omega^{-}(u)$ we denote the sets $\{x \in \Omega: u(x)>0\}$ and $\{x \in \Omega: u(x)<0\}$, respectively. Also, $\Lambda(u)$ denotes the set $\{x \in \Omega: u(x)=0\}$. 
The regularity of the solution, the Hausdorff dimension and the regularity of the free boundary are discussed in [2-5]. In [5], on the basis of the monotonicity formula due to Alt, Caffarelli, and Friedman, the boundedness of the second-order derivatives $D^{2} u$ of solutions to the two-phase membrane problem is proved. Moreover, in [3], a complete characterization of the global two-phase solution satisfying a quadratic growth at a two-phase free boundary point and at infinity is given. In [4] it has been shown that if $\lambda^{+}$and $\lambda^{-}$are Lipschitz, then, in two dimensions, the free boundary in a neighborhood of each branch point is the union of two $C^{1}$-graphs. Also, in higher dimensions, the free boundary has finite $(n-1)$-dimensional Hausdorff measure. Numerical approximation for the two-phase problem is discussed in [6].

In this article, by perturbation we mean the perturbation of the coefficients $\lambda^{+}$and $\lambda^{-}$ and the perturbation of the boundary values $g$. The case of the one phase obstacle problem has been studied in [7].

For given $\left(\lambda^{+}, \lambda^{-}\right) \in C^{0,1}(\Omega) \times C^{0,1}(\Omega)$, Equation 1.2 has a unique solution $u \in W_{\text {loc }}^{2, p}(\Omega)$ for

$1<p<\infty$ (see [8]). Define the map

$$
T:\left(\lambda^{+}, \lambda^{-}\right) \mapsto u,
$$

where $u$ is the solution of (1.2) corresponding to the coefficients $\lambda^{+}$and $\lambda^{-}$. The main results in this paper are the following:

1. The stability of solution with respect to boundary value and coefficients is shown.

2. Let $\bar{\lambda}=\left(\lambda^{+}, \lambda^{-}\right), \bar{h}=\left(h_{1}, h_{2}\right)$. By $u^{\bar{\lambda}+\varepsilon \bar{h}}$, we mean the solution of problem (1.2) with coefficients $\left(\lambda^{+}+\varepsilon h_{1}\right)$ and $\left(\lambda^{-}+\varepsilon h_{2}\right)$. If we Consider the map $T:\left(\lambda^{+}, \lambda^{-}\right) \mapsto u$, for given parameters $\lambda^{+}$and $\lambda^{+}$and a fixed Dirichlet condition, then the Gateaux derivative of this map is characterized in $H_{0}^{1}$. More precisely, it is shown in Theorem 3.4 that

$$
\frac{u^{\bar{\lambda}+\varepsilon \bar{h}}-u^{\bar{\lambda}}}{\varepsilon} \rightarrow w^{\bar{\lambda}, \bar{h}} \text { in } H_{0}^{1}(\Omega) \text { as } \varepsilon \rightarrow 0,
$$

where

$$
\Delta w^{\bar{\lambda}, \bar{h}}=h_{1} \chi_{\left\{u^{\bar{\lambda}}>0\right\}}-h_{2} \chi_{\left\{u^{\bar{\lambda}}<0\right\}}+\frac{\left(\lambda^{+}+\lambda^{-}\right)}{\left|\nabla u^{\bar{\lambda}}\right|} w^{\bar{\lambda}, \bar{h}} \mathcal{H}^{n-1} L_{\Gamma^{\prime \prime}}\left(u^{\bar{\lambda}}\right)
$$

3. (Theorem 3.5) Assuming that all free boundary points are one-phase points (points such that $\nabla u=0$ ), a stability result for the free boundary in the flavor of [7] is proved which says that

$$
\begin{aligned}
& \frac{\chi_{\left\{u^{\bar{\lambda}}+\varepsilon \bar{h}>0\right\}}-\chi_{\left\{u^{\bar{\lambda}}>0\right\}}}{\varepsilon}--\frac{1}{\lambda+} \frac{\partial \delta}{\partial v_{1}} d \Gamma^{+}, \text {in } H^{-1}(\Omega) \text { as } \varepsilon \rightarrow 0, \\
& \frac{\chi_{\left\{u^{\bar{\lambda}}+\varepsilon \bar{h}<0\right\}}-\chi_{\left\{u^{\bar{\lambda}}<0\right\}}}{\varepsilon} \rightarrow \frac{1}{\lambda^{-}} \frac{\partial \delta}{\partial v_{2}} d \Gamma^{-}, \text {in } H^{-1}(\Omega) \text { as } \varepsilon \rightarrow 0 .
\end{aligned}
$$


Were $\Gamma^{ \pm}=\partial\{ \pm u(x)>0\} \cap \Omega$. The function $\delta$ is constructed as a solution of certain Dirichlet problem in $\left\{u^{\bar{\lambda}}>0\right\}$. The vector $v_{1}$ stands for the exterior unit normal vector to $\partial\left\{u^{\bar{\lambda}}>0\right\}$.

The structure of article is organized as follows. In the next section, stability of solution with respect to boundary value and coefficients is studied. In Section 3, we prove that the map $T$ is Lipschitz continuous (Theorem 3.1) and differentiable (Theorem 3.4).

\section{Preliminary analysis and stability results}

In this section, we state some lemmas which have been proved in the case of onephase obstacle problem (see [9]). The following proposition shows the stability in $L^{\infty}$ norm. In what follows, we will denote by $B_{r}\left(x_{0}\right)$ the ball of radius $r$ centered at $x_{0}$ and, for simplicity, we use the notation $B_{r}=B_{r}(0)$.

Proposition 2.1. Let $u_{i}$ for $i=1,2$ be the solution of the following problem

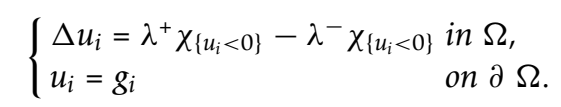

If $g_{1} \leq g_{2} \leq g_{1}+\varepsilon$, then $u_{1} \leq u_{2} \leq u_{1}+\varepsilon$. In particular,

$$
\left\|u_{2}-u_{1}\right\|_{L^{\infty}} \leq\left\|g_{1}-g_{2}\right\|_{L^{\infty}} .
$$

Proof. First, we show that $u_{1} \leq u_{2}$. Denote $\widetilde{\Omega}=\left\{x \in \Omega \mid u_{1}(x)>u_{2}(x)\right\}$; then, for all $x \in \tilde{\Omega}$ the following inequalities hold.

$$
\chi_{\left\{u_{1}>0\right\}} \geq \chi_{\left\{u_{2}>0\right\}}
$$

and

$$
\chi_{\left\{u_{1}<0\right\}} \leq \chi_{\left\{u_{2}<0\right\}} .
$$

These inequalities imply that

$$
\Delta u_{1}=\lambda^{+} \chi_{\left\{u_{1}>0\right\}}-\lambda^{-} \chi_{\left\{u_{1}<0\right\}} \geq \lambda^{+} \chi_{\left\{u_{2}>0\right\}}-\lambda^{-} \chi_{\left\{u_{2}<0\right\}}=\Delta u_{2}, \quad \text { in } \widetilde{\Omega},
$$

which shows that

$$
\Delta\left(u_{1}-u_{2}\right) \geq 0, \quad \forall x \in \tilde{\Omega} .
$$

One can see that on the boundary of $\widetilde{\Omega}$, the following holds:

$$
\left.\left(u_{1}-u_{2}\right)\right|_{\partial \widetilde{\Omega}}=\left\{\begin{array}{lr}
0 & x \in \partial \widetilde{\Omega} \backslash \partial \Omega \\
g_{1}-g_{2} & x \in \partial \Omega \backslash \partial \widetilde{\Omega} .
\end{array}\right.
$$

Note that by assumptions on $g_{1}$ and $g_{2}$, the inequality $u_{1}-u_{2} \leq 0$ will hold on the ә $\widetilde{\Omega}$. Thus, we have,

$$
\left\{\begin{array}{l}
\Delta\left(u_{1}-u_{2}\right) \geq 0 \text { in } \widetilde{\Omega} \\
\left(u_{1}-u_{2}\right) \leq 0 \text { on } \partial \widetilde{\Omega}
\end{array}\right.
$$

By maximum principle, we obtain that

$$
u_{1}-u_{2} \leq 0 \quad \forall x \in \widetilde{\Omega},
$$

which is impossible. Therefore, $\widetilde{\Omega}=\emptyset$. 
Let $u_{3}$ be the solution to the following problem:

$$
\begin{cases}\Delta u_{3}=\lambda^{+} \chi_{\left\{u_{3}>0\right\}}-\lambda^{-} \chi_{\left\{u_{3}<0\right\}} & \text { in } \Omega, \\ u_{3}=g_{1}+\varepsilon & \text { on } \partial \Omega .\end{cases}
$$

An analysis similar to the one above shows that if $v=u_{1}+\varepsilon-u_{3}$, then $v \geq 0$, which implies

$$
u_{1} \leq u_{2} \leq u_{3} \leq u_{1}+\varepsilon .
$$

$\square$

Lemma 2.2. Assume that $\inf _{B_{1}(0)} \lambda^{-}>\varepsilon>0$. Let $u$ be a solution to

$$
\Delta u=\lambda^{+} \chi_{\{u>0\}}-\lambda^{-} \chi_{\{u<0\}} \text { in } B_{1},
$$

and let $u^{\varepsilon}$ solve

$$
\Delta u^{\varepsilon}=\left(\lambda^{+}+\varepsilon\right) \chi_{\left\{u^{\varepsilon}>0\right\}}-\left(\lambda^{-}-\varepsilon\right) \chi_{\left\{u^{\varepsilon}<0\right\}} \text { in } B_{1},
$$

with $u=u^{\varepsilon}=g$ on $\partial B_{1}$. Then

$$
\left|u^{\varepsilon}-u\right| \leq C \varepsilon .
$$

Proof. Let $\varepsilon>0$,; we will show that $u^{\varepsilon} \leq u$. Set $D=\left\{x \in B_{1}: u^{\varepsilon}(x)>u(x)\right\}$. If $u^{\varepsilon} \leq 0$, on $D$, then $u<0$ on $D$ and $\Delta u=-\lambda^{-} \leq-\left(\lambda^{-}-\varepsilon\right) \leq \Delta u^{\varepsilon}$ : On the other hand, if $u^{\varepsilon}>0$; then $\Delta u^{\varepsilon}=\lambda^{+}+\varepsilon \geq \Delta u$. Therefore, $\Delta u^{\varepsilon} \geq \Delta u$ and, by maximum principle, $D=\varnothing$.

Now we claim that also $u+\varepsilon v \leq u^{\varepsilon}$ in $B_{1}$, where $v$ is the solution to $\Delta v=1$ with zero Dirichlet boundary data in $B_{1}$. Assume that

$$
\widetilde{\Omega}=\left\{x \in B_{1}: u+\varepsilon v>u^{\varepsilon}(x)\right\} .
$$

Note that $v(x) \leq 0$ in $B_{1}$, and so we have

$$
u^{\varepsilon}<u+\varepsilon v \leq u \text { in } \widetilde{\Omega} .
$$

Then, for all $x \in \tilde{\Omega}$, the following inequalities hold:

$$
\chi_{\{u>0\}} \geq \chi_{\left\{u^{\varepsilon}>0\right\}},
$$

and

$$
\chi_{\{u<0\}} \leq \chi_{\left\{u^{\varepsilon}<0\right\}} .
$$

In $\widetilde{\Omega}$, we have

$$
\begin{aligned}
& \Delta(u+\varepsilon v)=\Delta u+\varepsilon=\lambda^{+} \chi_{\{u>0\}}-\lambda^{-} \chi_{\{u<0\}}+\varepsilon \geq \lambda^{+} \chi_{\left\{u^{\varepsilon}>0\right\}}-\lambda^{-} \chi_{\left\{u^{\varepsilon}<0\right\}}+\varepsilon \\
& \geq\left(\lambda^{+}+\varepsilon\right) \chi_{\left\{u^{\varepsilon}>0\right\}}-\left(\lambda^{-}-\varepsilon\right) \chi_{\left\{u^{\varepsilon}<0\right\}}=\Delta u^{\varepsilon} .
\end{aligned}
$$

Therefore, we have

$$
\left\{\begin{array}{l}
\Delta\left(u+\varepsilon v-u^{\varepsilon}\right) \geq 0 \text { in } \quad \widetilde{\Omega}, \\
u+\varepsilon v-u^{\varepsilon}=0 \quad \text { on } \partial \widetilde{\Omega} .
\end{array}\right.
$$

This shows that $u+\varepsilon v \leq u^{\varepsilon}$ in $\widetilde{\Omega}$, which is impossible. Since

$$
v(x)=\frac{|x|^{2}-1}{2 n},
$$


this implies that $u^{\varepsilon} \geq-C \varepsilon+u$. Note that in the case when $\varepsilon<0$, with the assumption $\inf _{B_{1}(0)} \lambda^{+}>-\varepsilon>0$ one can prove that

$$
u \leq u^{\varepsilon} \leq u+\varepsilon v .
$$

$\square$

Remark 1. An analysis similar to Lemma 2.2 shows that if the coefficients $\lambda^{ \pm}$be perturbed by $\pm \varepsilon$, then $\left|u^{\varepsilon}-u\right| \leq C \varepsilon$.

Remark 2. The proofs of Proposition 2.1 and Lemma 2.2 show that if $u$ and $v$ solve the following problems, respectively:

$$
\begin{cases}\Delta u=\lambda_{1}^{+} \chi_{\{u>0\}}-\lambda_{1}^{-} \chi_{\{u<0\}} & \text { in } B_{1}, \\ u=g_{1} & \text { on } \partial B_{1},\end{cases}
$$

and

$$
\begin{cases}\Delta v=\lambda_{2}^{+} \chi_{\{v>0\}}-\lambda_{2}^{-} \chi_{\{v<0\}} & \text { in } B_{1} \\ v=g_{2} & \text { on } \partial B_{1}\end{cases}
$$

with $\lambda_{2}^{+} \geq \lambda_{1}^{+}, \lambda_{2}^{-} \leq \lambda_{1}^{-}, g_{2} \leq g_{1}$, then $u \geq v$. In particular,

$$
\Lambda(u) \subseteq \Lambda(v), \Omega^{+}(v) \subseteq \Omega^{+}(u) \text { and } \Omega^{-}(v) \subseteq \Omega^{-}(u) .
$$

Theorem 2.3. Let $u_{k}$ be a sequence of minimizer to (1.1), respectively with data $g_{k}$ and $\lambda_{k}^{ \pm}$, such that

$$
g_{k} \rightarrow g \text { in } H^{\frac{1}{2}}(\partial \Omega),
$$

and

$$
\lambda_{k}^{ \pm} \rightarrow \lambda^{ \pm} \quad \text { in } C^{0}(\Omega)
$$

Then,

$$
u_{k} \rightarrow u \text { in } H^{1}(\Omega)
$$

where $u$ is the minimizer of (1.1) with data $g$ and potential $\lambda^{ \pm}$.

Proof. First, one can see that $g$ is an admissible boundary data, i.e., $g$ changes sign on the boundary by the strong convergence of $g_{k}$ in $H^{\frac{1}{2}}(\partial \Omega)$. We denote by $u^{*}$ the solution to minimization problem (1.1) with data $g$ and $\lambda^{ \pm}$. Consider the minimum levels $c_{k}=I_{k}\left(u_{k}\right)$ and $c^{*}=I\left(u^{*}\right)$. Also the convergence of the boundary traces $g_{k}$ and of the $\lambda_{k}^{ \pm}$, ensures a bound on the sequence $c_{k}$. Since the sequence of functionals $\left\{I_{k}\right\}$ is uniformly coercive, from the fact that $I_{k}\left(u_{k}\right) \leq C$, we infer a bound on the sequence $\left\|u_{k}\right\|_{H^{1}(\Omega)}$; therefore, we can assume, up to a subsequence, that

$$
c_{k} \rightarrow c_{0} \text { and } u_{k} \rightarrow u \text { weakly in } H^{1}(\Omega) .
$$

Furthermore, by the weak continuity of the trace operator, we obtain

$$
\left.u\right|_{\partial \Omega}=g .
$$


The weak lower semi-continuity of the norm implies

$$
\int_{\Omega} \frac{1}{2}|\nabla u|^{2} d x \leq \operatorname{Lim} \inf \int_{\Omega} \frac{1}{2}\left|\nabla u_{k}\right|^{2} d x,
$$

and we also have

$$
\int_{\Omega}\left(\lambda^{+} \max (u, 0)-\lambda^{-} \min (u, 0)\right) d x \leq \operatorname{Lim} \inf \int_{\Omega}\left(\lambda_{k}^{+} \max \left(u_{k}, 0\right)-\lambda_{k}^{-} \min \left(u_{k}, 0\right)\right) d x .
$$

Note that the level

$$
c=\int_{\Omega}\left(\frac{1}{2}|\nabla u|^{2}+\lambda^{+} \max (u, 0)-\lambda^{-} \min (u, 0)\right) d x,
$$

is not necessarily a minimum, but, by the previous discussion it satisfies the inequalities

$$
c_{0} \geq c \geq c^{*} .
$$

We shall prove that $c_{0}=c^{*}$. Suppose, by contradiction, that $c^{*}<c_{0}$. Consider the harmonic extensions (denoted with the same notations) on $\Omega$ of $g_{i}$ 's and of $g$ and introduce

$$
h_{k}=g_{k}-g .
$$

Then, by construction

$$
\left\{\begin{array}{l}
h_{k} \rightarrow 0 \quad \text { in } H^{1}(\Omega) \\
\left.h_{k}\right|_{\partial \Omega} \rightarrow 0 \text { in } H^{\frac{1}{2}}(\partial \Omega) .
\end{array}\right.
$$

We define $w_{k}=u^{*}+h_{k}$, and observe that $\left.w_{k}\right|_{\partial \Omega}=g_{k}$. Moreover, by (1.7),

$$
w_{k} \rightarrow u^{*} \text { in } H^{1}(\Omega)
$$

Hence, it follows from the definition of $c_{k}$ that

$$
\int_{\Omega}\left(\frac{1}{2}\left|\nabla w_{k}\right|^{2}+\lambda_{k}^{+} \max \left(w_{k}, 0\right)-\lambda_{k}^{-} \min \left(w_{k}, 0\right)\right) d x \geq c_{k} .
$$

On the other hand, (1.8) gives

$$
\int_{\Omega}\left(\frac{1}{2}\left|\nabla w_{k}\right|^{2}+\lambda_{k}^{+} \max \left(w_{k}, 0\right)-\lambda_{k}^{-} \min \left(w_{k}, 0\right)\right) d x \rightarrow c^{*},
$$

which implies that $c^{*} \geq c_{0}$. Finally, from the equality of the minima $c_{0}=c=c^{*}$, we also deduce the strong convergence of $u_{k}$ in $H^{1}(\Omega)$.

\section{Perturbation formula for the free boundary}

In this section, we prove the continuity and differentiability of the map $T$. The case of one-phase obstacle problem was studied by Stojanovic [7].

Theorem 3.1. Assume $\lambda^{+}, \lambda^{-} \in L^{p}(\Omega)$ for $p>\frac{n}{2}$. The map $\left(\lambda^{+}, \lambda^{-}\right) \mapsto u$ is Lipschitz continuous in the following sense. If $u_{i}$ for $i=1,2$ solves

$$
\begin{cases}\Delta u_{i}=\lambda_{i}^{+} \chi_{\left\{u_{i}>0\right\}}-\lambda_{i}^{-} \chi_{\left\{u_{i}<0\right\}} & \text { in } \Omega, \\ u_{i}=g & \text { on } \partial \Omega,\end{cases}
$$


then

$$
\left\|u_{2}-u_{1}\right\|_{H^{1}(\Omega)} \leq C\left(\left\|\lambda_{1}^{+}-\lambda_{2}^{+}\right\|_{H^{-1}(\Omega)}+\left\|\lambda_{1}^{-}-\lambda_{2}^{-}\right\|_{H^{-1}(\Omega)}\right),
$$

and for $p>\frac{n}{2}$

$$
\left\|u_{2}-u_{1}\right\|_{L^{\infty}(\Omega)} \leq C\left(\left\|\lambda_{1}^{+}-\lambda_{2}^{+}\right\|_{L^{p}(\Omega)}+\left\|\lambda_{1}^{-}-\lambda_{2}^{-}\right\|_{L^{p}(\Omega)}\right) .
$$

We first prove the following lemma:

Lemma 3.2. If

$$
\left(\lambda_{1}^{+}-\lambda_{2}^{+}\right) \leq \varepsilon \in L^{p}, \quad p>n / 2, \varepsilon \geq 0, \text { and } \lambda_{1}^{-}=\lambda_{2}^{-},
$$

then

$$
u_{2}-u_{1} \leq \delta \in L^{\infty}(\Omega),
$$

where $\delta>0, \delta \in W^{2, p} \cap H_{0}^{1}$ solves

$$
\Delta \delta=-\varepsilon .
$$

Moreover, the same argument can be applied with

$$
\left(\lambda_{2}^{-}-\lambda_{1}^{-}\right) \leq \varepsilon \text { and } \lambda_{2}^{+}=\lambda_{1}^{+} .
$$

Proof. Let

$$
\begin{array}{lr}
\lambda_{3}^{+}=\lambda_{1}^{+} \chi_{\left\{u_{1}>0\right\},} & \lambda_{3}^{-}=\lambda_{1}^{-} \chi_{\left\{u_{1}<0\right\},} \\
\lambda_{4}^{+}=\min \left\{\lambda_{2}^{+}, \lambda_{3}^{+}\right\}, & \lambda_{4}^{-}=\lambda_{3}^{-} .
\end{array}
$$

Then, by the same proof as in the first part of Lemma 2.2, one gets

$$
u_{3}=u_{1}, \quad u_{4} \geq u_{2}
$$

where $u_{3}$ and $u_{4}$ solve Equation 1.2 with coefficients $\lambda_{3}^{ \pm}, \lambda_{4}^{ \pm}$, respectively. Relation (1.10) gives

$$
\left(\lambda_{3}^{+}-\lambda_{4}^{+}\right) \chi_{\left\{u_{1}>0\right\}} \leq \varepsilon .
$$

Also, by the choice of $\lambda_{4}^{+}$, we have

$$
\lambda_{4}^{+} \chi_{\left\{u_{1} \leq 0\right\}}=0, \quad \lambda_{4}^{-} \chi_{\left\{u_{1} \geq 0\right\}}=0 .
$$

We will show that

$$
\left(u_{4}-\left(u_{3}+\delta\right)\right)^{+}=0 .
$$

First, note that

$$
\begin{gathered}
\Delta u_{4}=\lambda_{4}^{+} \chi_{\left\{u_{4}>0\right\}}-\lambda_{4}^{-} \chi_{\left\{u_{4}<0\right\}}, \\
\Delta\left(u_{3}+\delta\right)=\lambda_{3}^{+} \chi_{\left\{u_{3}>0\right\}}-\lambda_{3}^{-} \chi_{\left\{u_{3}<0\right\}}-\varepsilon .
\end{gathered}
$$

Therefore,

$$
\Delta\left(u_{4}-\left(u_{3}+\delta\right)\right)=\lambda_{4}^{+} \chi_{\left\{u_{4}>0\right\}}-\lambda_{4}^{-} \chi_{\left\{u_{4}<0\right\}}-\lambda_{3}^{+} \chi_{\left\{u_{3}>0\right\}}+\lambda_{3}^{-} \chi_{\left\{u_{3}<0\right\}}+\varepsilon .
$$


Rearranging the above terms gives

$$
\begin{aligned}
& \Delta\left(u_{4}-\left(u_{3}+\delta\right)\right)-\lambda_{4}^{+} \chi_{\left\{u_{4}>0\right\}}+\lambda_{4}^{+} \chi_{\left\{u_{3}>0\right\}}+\lambda_{4}^{-} \chi_{\left\{u_{4}<0\right\}}-\lambda_{3}^{-} \chi_{\left\{u_{3}<0\right\}} \\
& =\left(\lambda_{4}^{+}-\lambda_{3}^{+}\right) \chi_{\left\{u_{3}>0\right\}}+\varepsilon \geq 0 .
\end{aligned}
$$

Multiplying by $\left(u_{4}-\left(u_{3}+\delta\right)\right)^{+}$and integrating by parts gives

$$
\begin{aligned}
0 \leq \int_{\Omega}\left[\left(u_{4}-\right.\right. & \left.\left.\left(u_{3}+\delta\right)\right)^{+} \Delta\left(u_{4}-\left(u_{3}+\delta\right)\right)\right] d x \\
& -\int_{\Omega}\left[\lambda_{4}^{+}\left(\chi_{\left\{u_{4}>0\right\}}-\chi_{\left\{u_{3}>0\right\}}\right)-\lambda_{4}^{-} \chi_{\left\{u_{4}<0\right\}}+\lambda_{3}^{-} \chi_{\left\{u_{3}<0\right\}}\right]\left(u_{4}-\left(u_{3}+\delta\right)\right)^{+} d x .
\end{aligned}
$$

Then,

$$
\begin{aligned}
& -\int_{\Omega} \mid \nabla\left(\left.\left(u_{4}-\left(u_{3}+\delta\right)\right)^{+}\right|^{2} d x\right. \\
& -\int_{\Omega}\left[\lambda_{4}^{+}\left(\chi_{\left\{u_{4}>0\right\}}-\chi_{\left\{u_{3}>0\right\}}\right)-\lambda_{4}^{-} \chi_{\left\{u_{4}<0\right\}}+\lambda_{3}^{-} \chi_{\left\{u_{3}<0\right\}}\right]\left(u_{4}-\left(u_{3}+\delta\right)\right)^{+} d x \geq 0 .
\end{aligned}
$$

It follows that

$$
\begin{aligned}
& \int_{\Omega} \mid \nabla\left(\left.\left(u_{4}-\left(u_{3}+\delta\right)\right)^{+}\right|^{2} d x\right. \\
& +\int_{\left\{u_{4}-\left(u_{3}+\delta\right)>0\right\}}\left[\lambda_{4}^{+} \chi_{\left\{u_{4}>0\right\}}-\chi_{\left\{u_{3}>0\right\}}-\lambda_{4}^{-} \chi_{\left\{u_{4}<0\right\}}+\lambda_{3}^{-} \chi_{\left\{u_{3}<0\right\}}\right]\left(u_{4}-\left(u_{3}+\delta\right)\right) d x \leq 0 .
\end{aligned}
$$

Note that

$$
\left[\lambda_{4}^{+}\left(\chi_{\left\{u_{4}>0\right\}}-\chi_{\left\{u_{3}>0\right\}}\right)-\lambda_{4}^{-} \chi_{\left\{u_{4}<0\right\}}+\lambda_{3}^{-} \chi_{\left\{u_{3}<0\right\}}\right]\left(u_{4}-u_{3}\right) \geq 0 .
$$

Then, we have

$$
\begin{aligned}
& \int_{\Omega} \mid \nabla\left(\left.\left(u_{4}-\left(u_{3}+\delta\right)\right)^{+}\right|^{2} d x\right. \\
& -\int_{\left\{u_{4}-\left(u_{3}+\delta\right)>0\right\}}\left[\lambda_{4}^{+} \chi_{\left\{u_{4}>0\right\}}-\chi_{\left\{u_{3}>0\right\}}-\lambda_{3}^{-}\left(\chi_{\left\{u_{4}<0\right\}}-\chi_{\left\{u_{3}<0\right\}}\right] \delta d x \leq 0 .\right.
\end{aligned}
$$

However,

$$
\begin{aligned}
& \int_{\left\{u_{4}-\left(u_{3}+\delta\right)>0\right\}}\left[\lambda_{4}^{+}\left(\chi_{\left\{u_{4}>0\right\}}-\chi_{\left\{u_{3}>0\right\}}\right)-\lambda_{4}^{-}\left(\chi_{\left\{u_{4}<0\right\}}-\chi_{\left\{u_{3}<0\right\}}\right)\right] \delta d x \\
& =\int_{\left\{u_{4}-\left(u_{3}+\delta\right)>0\right\}} \lambda_{4}^{+}\left(\chi_{u_{4}>0} \chi_{u_{3} \leq 0}\right) \delta d x-\int_{\left\{u_{4}-\left(u_{3}+\delta\right)>0\right\}} \lambda_{4}^{-}\left(\chi_{\left\{u_{4}<0\right\}} \chi_{\left\{u_{3} \geq 0\right\}}\right) \delta d x=0 .
\end{aligned}
$$

In the last equation, we have used (1.16).

口

Thus we completed the proof of Theorem 3.1.

Proof of Theorem 3.1. By elliptic regularity and Lemma 3.2, we have

$$
\delta \in W_{1 \mathrm{oc}}^{2, p}(\Omega) \cap H_{0}^{1}(\Omega),
$$

and, consequently, the Sobolev embedding $W_{\mathrm{loc}}^{2, p} \hookrightarrow C_{\mathrm{loc}}^{0, \frac{n}{2 p}}$ for $p>\frac{n}{2}$, implies

$$
\delta \in C^{0, \alpha}(\Omega) \text {, with } 0<\alpha<1 .
$$


Therefore,

$$
\|\delta\|_{L^{\infty}} \leq C\|\varepsilon\|_{L^{p}} .
$$

Now if we assume $\left|\lambda_{1}^{+}-\lambda_{2}^{+}\right| \leq \varepsilon$, then it will follows that $\left|u_{2}-u_{1}\right|<\delta$. To complete the proof, assume that

$$
\left(\lambda_{1}^{+}-\lambda_{2}^{+}\right) \leq \varepsilon \text { and }\left(\lambda_{2}^{-}-\lambda_{1}^{-}\right) \leq \varepsilon .
$$

Set $\Delta u^{\prime}=\lambda_{2}^{+} \chi_{\left\{u^{\prime}>0\right\}}-\lambda_{1}^{-} \chi_{\left\{u^{\prime}<0\right\}}$. Then, we have

$$
u_{2}-u_{1}=u_{2}-u^{\prime}+u^{\prime}-u_{1} \leq 2 \delta,
$$

and

$$
\left\|u_{2}-u_{1}\right\|_{L^{\infty}} \leq\left\|u_{2}-u^{\prime}\right\|_{L^{\infty}}+\left\|u^{\prime}-u_{1}\right\|_{L^{\infty}} .
$$

By Equation 1.11, we obtain

$$
\left\|u_{2}-u_{1}\right\|_{L^{\infty}} \leq C\left(\left\|\lambda_{1}^{+}-\lambda_{2}^{+}\right\|_{L^{p}}+\left\|\lambda_{1}^{-}-\lambda_{2}^{-}\right\|_{L^{p}}\right) .
$$

$\square$

The proof of Theorem 3.4 uses the following theorem, proved by I. Blank in [9].

Theorem 3.3. (Linear Stability of the Free Boundary in the one phase case). Suppose that the free boundary is locally uniformly $C^{1, \alpha}$ regular in $B_{1}$. Let $w, w^{\varepsilon}$ be the solutions of the following one-phase problems, respectively,

$$
\left\{\begin{array}{lll}
\Delta w=\lambda^{+} \chi\{w>0\} & \text { in } & B_{1} \\
w=g & \text { on } & \partial B_{1}
\end{array}\right.
$$

and

$$
\left\{\begin{array}{lll}
\Delta w^{\varepsilon}=\left(\lambda^{+}+\varepsilon\right) \chi_{\left\{w^{\varepsilon}>0\right\}} & \text { in } & B_{1} \\
w^{\varepsilon}=g & \text { on } & \partial B_{1}
\end{array} .\right.
$$

Then, for \& small enough, we have

$$
\operatorname{dist}\left(\Gamma(w), \Gamma\left(w^{\varepsilon}\right)\right) \leq C \varepsilon, \quad \text { on } \quad B_{\frac{1}{2}} .
$$

Remark 3. The analogue of Theorem 3.3 can be proved for the two-phase membrane problem in the following cases:

(1) When all the points are regular one-phase points (cf. Theorem 3.3).

(2) When all the points are two-phase points with $|\nabla u|=0$ (branching points).

(3) When $|\nabla u|$ is uniformly bounded from below (cf. Estimate 1.19).

Although we could not prove this theorem for the two-phase case in general, there are grounds, however, to suggest that it holds true in this case as well.

The proof of part (3) is as follows. Suppose $\varepsilon>0, h_{1}>0, h_{2}<0$ and $\inf _{\Omega} \lambda^{-}>-\varepsilon h_{2}$. Then Lemma 2.2 implies that

$$
-C \varepsilon+u^{\bar{\lambda}} \leq u^{\bar{\lambda}+\varepsilon \bar{h}} \leq u^{\bar{\lambda}} .
$$


Also, $u^{\bar{\lambda}} \geq C^{\prime} \operatorname{dist}\left(x, \Gamma^{\prime \prime}\left(u^{\bar{\lambda}}\right)\right)$ for $x \in \Omega^{+} \cap B_{r}$ where $r$ is small enough, which gives

$$
u^{\bar{\lambda}+\varepsilon \bar{h}} \geq-C \varepsilon+C^{\prime} \operatorname{dist}\left(x, \Gamma^{\prime \prime}\left(u^{\bar{\lambda}}\right)\right) \text {. }
$$

Thus, $u^{\bar{\lambda}+\varepsilon \bar{h}}$ is positive provided that $\operatorname{dist}\left(x, \Gamma^{\prime \prime}\left(u^{\bar{\lambda}}\right)\right) \geq C^{\prime \prime} \varepsilon$, which shows

$$
\operatorname{dist}\left(\Gamma^{\prime \prime}\left(u^{\bar{\lambda}}\right), \Gamma^{\prime \prime}\left(u^{\bar{\lambda}+\varepsilon \bar{h}}\right)\right) \leq C_{1} \varepsilon .
$$

Now we shall prove that the map $\left(\lambda^{+}, \lambda^{-}\right) \mapsto u^{\bar{\lambda}}$ is differentiable in the following sense:

Theorem 3.4. The mapping

$$
T: C^{0,1}(\Omega) \times C^{0,1}(\Omega) \rightarrow W^{2, p}(\Omega),
$$

defined by $u=T\left(\lambda^{+}, \lambda^{-}\right)$is differentiable. Furthermore, if $\bar{\lambda}, \bar{h} \in C^{0,1}(\Omega) \times C^{0,1}(\Omega)$.

Then, there exists $w^{\bar{\lambda}, \bar{h}} \in H_{0}^{1}$, such that

$$
\frac{u^{\bar{\lambda}+\varepsilon \bar{h}}-u^{\bar{\lambda}}}{\varepsilon} \rightarrow w^{\bar{\lambda}, \bar{h}} \text { in } H_{0}^{1}(\Omega) \text { as } \varepsilon \rightarrow 0
$$

where

$$
\Delta w^{\bar{\lambda}, \bar{h}}=h_{1} \chi_{\left\{u^{\bar{\lambda}}>0\right\}}-h_{2} \chi_{\left\{u^{\bar{\lambda}}<0\right\}}+\frac{\left(\lambda^{+}+\lambda^{-}\right)}{\left|\nabla u^{\bar{\lambda}}\right|} w^{\bar{\lambda}, \bar{h}} \mathcal{H}^{n-1} L_{\Gamma^{\prime \prime}\left(u^{\bar{\lambda}}\right)} .
$$

In Equation 1.20, $\mathcal{H}^{n-1}$ denotes the $(n-1)$-dimensional Hausdorff measure.

Proof. We have

$$
\Delta u^{\bar{\lambda}}=\lambda^{+} \chi_{\left\{u^{\bar{\lambda}}>0\right\}}-\lambda^{-} \chi_{\left\{u^{\bar{\lambda}}<0\right\}}
$$

and

$$
\Delta u^{\bar{\lambda}+\varepsilon \bar{h}}=\left(\lambda^{+}+\varepsilon h_{1}\right) \chi_{\left\{u^{\bar{x}+\varepsilon \bar{h}}>0\right\}}-\left(\lambda^{-}+\varepsilon h_{2}\right) \chi_{\left\{u^{\overline{\bar{x}}+\varepsilon \bar{h}}<0\right\}} .
$$

Therefor,

$$
\begin{aligned}
\Delta\left(u^{\bar{\lambda}+\varepsilon \bar{h}}-u^{\bar{\lambda}}\right)=\lambda^{+}\left(\chi_{\left\{u^{\bar{\lambda}}+\varepsilon \bar{h}>0\right\}}-\chi_{\left\{u^{\bar{\lambda}}>0\right\}}\right)+\lambda^{-}\left(\chi_{\left\{u^{\overline{\bar{\lambda}}}<0\right\}}\right. & \\
& \left.-\chi_{\left\{u^{\overline{\bar{\lambda}}+\varepsilon \bar{h}}<0\right\}}\right)+\varepsilon h_{1} \chi_{\left\{u^{\overline{\bar{\lambda}}}+\varepsilon \overline{\bar{h}}>0\right\}}-\varepsilon h_{2} \chi_{\left\{u^{\bar{\lambda}}+\varepsilon \bar{h}<0\right\}} .
\end{aligned}
$$

We multiply both sides of (1.21) by $\left(u^{\bar{\lambda}+\varepsilon \bar{h}}-u^{\bar{\lambda}}\right)$ and integrate by parts and we obtain

$$
\begin{aligned}
\int_{\Omega}\left|\nabla\left(u^{\bar{\lambda}+\varepsilon \bar{h}}-u^{\bar{\lambda}}\right)\right|^{2} d x= & -\int_{\Omega} \lambda^{+}\left(\chi_{\left\{u^{\bar{\lambda}+\varepsilon \bar{h}}>0\right\}}-\chi_{\left\{u^{\bar{\lambda}}>0\right\}}\right)\left(u^{\bar{\lambda}+\varepsilon \bar{h}}-u^{\bar{\lambda}}\right) d x \\
& +\int_{\Omega} \lambda^{-}\left(\chi_{\left\{u^{\bar{\lambda}+\varepsilon \bar{h}}<0\right\}}-\chi_{\left\{u^{\bar{\lambda}}<0\right\}}\right)\left(u^{\bar{\lambda}+\varepsilon \bar{h}}-u^{\bar{\lambda}}\right) d x \\
& -\int_{\Omega} \varepsilon\left(h_{1} \chi_{\left\{u^{\overline{\bar{\lambda}}+\varepsilon \bar{h}}>0\right\}}-h_{2} \chi_{\left\{u^{\bar{\lambda}+\varepsilon \bar{h}}<0\right\}}\right)\left(u^{\bar{\lambda}+\varepsilon \bar{h}}-u^{\bar{\lambda}}\right) d x .
\end{aligned}
$$

Note that

$$
\left(\chi_{\left\{u^{\bar{\lambda}+\varepsilon \bar{h}}>0\right\}}-\chi_{\left\{u^{\bar{\lambda}}>0\right\}}\right)\left(u^{\bar{\lambda}+\varepsilon \bar{h}}-u^{\bar{\lambda}}\right) \geq 0,
$$


and

$$
\left(\chi_{\left\{u^{\bar{\lambda}+\varepsilon \bar{h}}<0\right\}}-\chi_{\left\{u^{\bar{\lambda}}<0\right\}}\right)\left(u^{\bar{\lambda}+\varepsilon \bar{h}}-u^{\bar{\lambda}}\right) \leq 0 .
$$

Therefore,

$$
\int_{\Omega}\left|\nabla\left(u^{\bar{\lambda}+\varepsilon \bar{h}}-u^{\bar{\lambda}}\right)\right|^{2} d x \leq \int_{\Omega} \varepsilon\left(h_{1} \chi_{\left\{u^{\bar{\lambda}+\varepsilon \bar{h}}>0\right\}}-h_{2} \chi_{\left\{u^{\bar{\lambda}+\varepsilon \bar{h}}<0\right\}}\right)\left(u^{\bar{\lambda}+\varepsilon \bar{h}}-u^{\bar{\lambda}}\right) d x .
$$

The Hölder inequality implies

$$
\begin{gathered}
\left\|\nabla\left(u^{\bar{\lambda}+\varepsilon \bar{h}}-u^{\bar{\lambda}}\right)\right\|_{L^{2}(\Omega)}^{2} \leq \varepsilon\left\|h_{1} \chi_{\left\{u^{\bar{\lambda}+\varepsilon \bar{h}}>0\right\}}-h_{2} \chi_{\left\{u^{\overline{\bar{\lambda}}+\overline{\bar{h}}}<0\right\}}\right\|_{L^{2}(\Omega)}\left\|u^{\bar{\lambda}+\varepsilon \bar{h}}-u^{\bar{\lambda}}\right\|_{L^{2}(\Omega)} \\
\leq \varepsilon\left(\left\|h_{1}\right\|_{L^{2}(\Omega)}+\left\|h_{2}\right\|_{L^{2}(\Omega)}\right)\left\|u^{\bar{\lambda}+\varepsilon \bar{h}}-u^{\bar{\lambda}}\right\|_{L^{2}(\Omega)} .
\end{gathered}
$$

Moreover, by the Poincaré inequality, we have

$$
\left\|\nabla\left(\frac{u^{\bar{\lambda}+\varepsilon \bar{h}}-u^{\bar{\lambda}}}{\varepsilon}\right)\right\|_{L^{2}(\Omega)} \leq C\left(\left\|h_{1}\right\|_{L^{2}(\Omega)}+\left\|h_{2}\right\|_{L^{2}(\Omega)}\right) .
$$

From (1.22), the weak convergence to a limit, denoted by $w^{\bar{\lambda}, \bar{h}}$, follows (for a subsequence). Here, we show that $w^{\bar{\lambda}, \bar{h}}$ satisfies (1.20). Multiply (1.21) by a test function $\varphi$, where $\varphi$ has compact support in $\left\{u^{\bar{\lambda}}>0\right\}$, and then divide by $\varepsilon$,

$$
-\int_{\Omega} \nabla\left(\frac{u^{\bar{\lambda}+\varepsilon \bar{h}}-u^{\bar{\lambda}}}{\varepsilon}\right) \cdot \nabla \phi d x=\int_{\Omega} \frac{\lambda^{+}}{\varepsilon}\left(\chi_{\left\{u^{\bar{\lambda}+\varepsilon \bar{h}}>0\right\}}-\chi_{\left\{u^{\bar{\lambda}}>0\right\}}\right) \phi d x+\int_{\Omega} h_{1} \chi_{\left\{u^{\bar{\lambda}}>0\right\}} \phi d x(1.23)
$$

Assume that $d$ is the distance between $\operatorname{supp}(\varphi)$ and $\Gamma^{+}\left(u^{\bar{\lambda}}\right)$. If $u^{\bar{\lambda}}(x) \geq c d^{2}$, then, (since $u^{\bar{\lambda}+\varepsilon \bar{h}} \rightarrow u^{\bar{\lambda}}$ ) for $\varepsilon$ small enough, we have

$$
\left|u^{\bar{\lambda}+\varepsilon \bar{h}}(x)-u^{\bar{\lambda}}(x)\right| \leq \frac{c d^{2}}{2}
$$

and so $u^{\bar{\lambda}+\varepsilon \bar{h}}(x) \geq \frac{c d^{2}}{2}>0$. This means that, for each $\varphi$, one can chose $\varepsilon$ small enough such that

$$
\left(\chi_{\left\{u^{\bar{\lambda}+\varepsilon \bar{h}}>0\right\}}-\chi_{\left\{u^{\bar{\lambda}}>0\right\}}\right)=0 \text { in } \operatorname{supp} \phi .
$$

In particular, passing to the limit in (1.23), we obtain that in the set $\left\{u^{\bar{\lambda}}>0\right\}$, equation

$$
\Delta w^{\bar{\lambda}, \bar{h}}=h_{1}
$$

holds. Similarly, in the set $\left\{u^{\bar{\lambda}}>0\right\}$, one has

$$
\Delta w^{\bar{\lambda}, \bar{h}}=-h_{2}
$$

Now let $x_{0}$ be a one-phase regular point for $u^{\bar{\lambda}}$ and $x_{\varepsilon} \in \Gamma\left(u^{\bar{\lambda}+\varepsilon \bar{h}}\right)$ where $x_{\varepsilon}$ has minimal distance to $x_{0}$.

Assumption In what follows, we assume that the estimate (1.18) in Theorem 3.3 also holds for one-phase points in our case. A straightforward calculation gives 


$$
\begin{aligned}
& \lim _{\varepsilon \rightarrow 0} \frac{u^{\bar{\lambda}+\varepsilon \bar{h}}\left(x_{0}\right)-u^{\bar{\lambda}}\left(x_{0}\right)}{\varepsilon} \\
& =\lim _{\varepsilon \rightarrow 0} \frac{u^{\bar{\lambda}+\varepsilon \bar{h}}\left(x_{\varepsilon}\right)+\left(x_{\varepsilon}-x_{0}\right)^{T} \cdot \nabla u^{\bar{\lambda}+\varepsilon \bar{h}}\left(x_{\varepsilon}\right)+O\left(\left(x_{\varepsilon}-x_{0}\right)^{2}\right)-u^{\bar{\lambda}}\left(x_{0}\right)}{\varepsilon} \\
& =\lim _{\varepsilon \rightarrow 0} \frac{\left(x_{\varepsilon}-x_{0}\right)^{T} \cdot \nabla u^{\bar{\lambda}+\varepsilon \bar{h}}\left(x_{\varepsilon}\right)}{\varepsilon}=0,
\end{aligned}
$$

which shows that $w^{\bar{\lambda}, \bar{h}}=0$ at one-phase regular points.

To complete the proof, let us assume that $x_{0} \in \Gamma^{\prime \prime}\left(u^{\bar{\lambda}}\right)$. Let $v$ denote the normal to the free boundary $\Gamma^{\prime \prime}\left(u^{\bar{\lambda}}\right)$ at $x_{0}$, that is $v=\frac{\nabla u\left(x_{0}\right)}{\left|\nabla u\left(x_{0}\right)\right|}$. Assume that $B_{r}\left(x_{0}\right)$ is a ball centered at $x_{0}$ where $r$ is small enough. Since $\nabla u\left(x_{0}\right) \neq 0$, then $\Gamma^{\prime \prime}\left(u^{\bar{\lambda}}\right)$ can be represented as $\left(x^{\prime}\right.$, $\left.f\left(x^{\prime}\right)\right)$ where $f$ is a $C^{1, \alpha}$ graph. We have

$$
v=e_{n}+O\left(r^{\alpha}\right) .
$$

Let $\Omega_{\varepsilon}$ be the region between $\Gamma^{\prime \prime}\left(u^{\bar{\lambda}}\right)$ and $\Gamma^{\prime \prime}\left(u^{\bar{\lambda}+\varepsilon \bar{h}}\right)$. From (1.21) we obtain

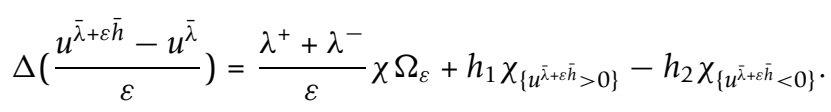

The term $\frac{1}{\varepsilon} \chi_{\Omega_{\varepsilon}}$ converges weakly as $\varepsilon \rightarrow 0$, to a measure $\mu$ with support on $\Gamma^{\prime \prime}(u)$. For any ball $B_{r}\left(x_{0}\right)$ with $x_{0} \in \Gamma^{\prime \prime}(u)$, set

$$
\mu\left(B_{r}\right)=\lim _{\varepsilon \rightarrow 0} \int_{B_{r}} \frac{1}{\varepsilon} \chi_{\Omega_{\varepsilon}} d x .
$$

Estimate (1.19) shows that $\mu$ is a finite measure, since

$$
\mu\left(B_{r}\right)=\lim _{\varepsilon \rightarrow 0} \int_{B_{r} \cap \Omega_{\varepsilon}} \frac{1}{\varepsilon} d x=\lim _{\varepsilon \rightarrow 0} \frac{\left|B_{r} \cap \Omega_{\varepsilon}\right|}{\varepsilon} \leq C .
$$

We want to prove that

$$
\lim _{r \rightarrow 0} \frac{\mu\left(B_{r}\left(x_{0}\right)\right)}{\mathcal{H}^{n-1}\left\lfloor\Gamma^{\prime \prime}(u)\left(B_{r}\left(x_{0}\right)\right)\right.}=\frac{w^{\bar{\lambda}, \bar{h}}\left(x_{0}\right)}{\left|\nabla u^{\bar{\lambda}}\left(x_{0}\right)\right|} .
$$

Then, $\mu$ can be written as (see [10], Chapter I)

$$
\mu=\lim _{r \rightarrow 0} \frac{\mu\left(B_{r}\right)}{\mathcal{H}^{n-1} \bigsqcup_{\Gamma^{\prime \prime}}\left(u^{\bar{\lambda}}\right)\left(B_{r}\right)} \cdot \mathcal{H}^{n-1} .
$$

Let $d$ be the distance of $x_{0}$ to $\Gamma^{\prime \prime}\left(u^{\bar{\lambda}+\varepsilon \bar{h}}\right)$ in direction of $v$, using Taylor expansion, we get

$$
d=\frac{u^{\bar{\lambda}+\varepsilon \bar{h}}\left(x_{0}\right)}{\left|\nabla u^{\bar{\lambda}+\varepsilon \bar{h}}\left(x_{\varepsilon}\right)\right|}+O(\varepsilon)
$$


In order to show (1.25), we have

$$
\begin{aligned}
\mu\left(B_{r}\right)= & \lim _{\varepsilon \rightarrow 0} \int_{B_{r}} \frac{1}{\varepsilon} \chi_{\Omega_{\varepsilon}} d x=\lim _{\varepsilon \rightarrow 0} \frac{1}{\varepsilon} d\left|B_{r}^{\prime}\right|+O\left(r^{n-1}\right)=\quad \text { by (1.26) } \\
& =\lim _{\varepsilon \rightarrow 0} \frac{u^{\bar{\lambda}+\varepsilon \bar{h}}\left(x_{0}\right)-u^{\bar{\lambda}}\left(x_{0}\right)}{\varepsilon} \frac{1}{\left|\nabla u^{\bar{\lambda}+\varepsilon \bar{h}}\left(x_{\varepsilon}\right)\right|}\left|B_{r}^{\prime}\right|+O\left(r^{n-1}\right) \\
& =\frac{w^{\bar{\lambda}, \bar{h}}\left(x_{0}\right)}{\left|\nabla u^{\bar{\lambda}}\left(x_{0}\right)\right|}\left|B_{r}^{\prime}\right|+O\left(r^{n-1}\right),
\end{aligned}
$$

where $\left|B_{r}^{\prime}\right|$ is the measure of $B_{r}^{\prime}=B_{r} \cap\left\{x_{n}=0\right\}$. In addition, we have

$$
\int_{B_{r}} d \mathcal{H}^{n-1}\left\lfloor_{\Gamma^{\prime \prime}\left(u^{\bar{\lambda}}\right)}=\int_{B_{r}^{\prime}} \sqrt{1+|\nabla f|^{2}}=\left|B_{r}^{\prime}\right|+r^{n-1} O\left(r^{2 \alpha}\right) .\right.
$$

Therefore,

$$
\lim _{r \rightarrow 0} \frac{\mu\left(B_{r}\right)}{\int_{B_{r}} d \mathcal{H}^{n-1} \bigsqcup_{\Gamma^{\prime \prime}\left(u^{\bar{\lambda}}\right)}}=\lim _{r \rightarrow 0} \frac{\frac{w^{\bar{\lambda}, \bar{h}}\left(x_{0}\right)}{\left|\nabla u^{\bar{\lambda}}\left(x_{0}\right)\right|}\left|B_{r}^{\prime}\right|}{\left|B_{r}^{\prime}\right|}=\frac{w^{\bar{\lambda}, \bar{h}}\left(x_{0}\right)}{\left|\nabla u^{\bar{\lambda}}\left(x_{0}\right)\right|} .
$$

We deduce that, $w^{\bar{\lambda}, \bar{h}} \in H_{0}^{1}(\Omega)$ satisfies (1.20).

口

Remark 4. If for all free boundary points $\nabla u=0$, which means that $\Gamma(u)=\Gamma^{\prime}(u)$, then

$$
w^{\bar{\lambda}, \bar{h}}= \begin{cases}\delta^{\bar{\lambda}, \bar{h}} \text { in }\left\{u^{\bar{\lambda}}>0\right\} \\ 0 \text { in }\left\{u^{\bar{\lambda}}=0\right\} \\ \delta^{\bar{\lambda}, \bar{h}} \text { in }\left\{u^{\bar{\lambda}}<0\right\}\end{cases}
$$

where $\delta^{\bar{\lambda}, \bar{h}}$ is the unique solution of the elliptic equation

$$
\begin{cases}\Delta \delta=h_{1} & \text { in }\left\{u^{\bar{\lambda}}>0\right\} \\ \delta=0 & \text { on } \partial\left\{u^{\bar{\lambda}}>0\right\} \\ \Delta \delta=-h_{2} & \text { in }\left\{u^{\bar{\lambda}}<0\right\} \\ \delta=0 & \text { on } \partial\left\{u^{\bar{\lambda}}<0\right\}\end{cases}
$$

Remark 5. Consider the following two-phase problem in dimension one $(n=1)$, where $\lambda_{1}, \lambda_{2}$ are constants.

$$
\left\{\begin{array}{l}
u^{\prime \prime}=\lambda_{1} \chi_{\{u>0\}}-\lambda_{2} \chi_{\{u<0\}} \quad \operatorname{in}(-1,+1) \\
u(-1)=a<0, \quad u(+1)=b>0 .
\end{array}\right.
$$

Straightforward calculations show that if $\sqrt{\frac{b-a}{\lambda_{1}}}+\sqrt{\frac{b-a}{\lambda_{2}}} \leq 2$, then the set $\{x \in \Omega: u(x)$ $=0\}$ has a positive measure. In this setting, an interesting question is which conditions in higher dimensions will imply that the zero set has positive measure in $B_{1}$.

Example 1 Let $\bar{\lambda}=(4,2), \bar{h}=(1,1)$. Consider the equation

$$
\left\{\begin{array}{l}
u^{\prime \prime}=4 \chi_{\{u>0\}}-2 \chi_{\{u<0\}} \\
u(+1)=+1, u(-1)=-1
\end{array}\right.
$$


One can obtain

$$
u^{\lambda+\varepsilon h}=\left\{\begin{array}{lr}
\left(2+\frac{\varepsilon}{2}\right) x^{2}-(4+\varepsilon)\left(1-\sqrt{\frac{2}{4+\varepsilon}}\right) x & 1-\sqrt{\frac{2}{4+\varepsilon}} \leq x \leq 1, \\
-\left(1+\frac{\varepsilon}{2}\right)+(4+\varepsilon)\left(1-\sqrt{\frac{2}{4+\varepsilon}}\right) & \\
0 & -1+\sqrt{\frac{2}{2+\varepsilon}} \leq x \leq 1-\sqrt{\frac{2}{4+\varepsilon}} \\
\left(-1-\frac{\varepsilon}{2}\right) x^{2}-(2+\varepsilon)\left(-1+\sqrt{\frac{2}{2+\varepsilon}}\right) x & -1 \leq x \leq-1+\sqrt{\frac{2}{2+\varepsilon}} . \\
+\left(\frac{\varepsilon}{2}\right)+\sqrt{\frac{2}{2+\varepsilon}}+(2+\varepsilon)\left(-1+\sqrt{\frac{2}{2+\varepsilon}}\right) &
\end{array}\right.
$$

Consequently, one computes

$$
\lim _{\varepsilon \rightarrow 0} \frac{u^{\bar{\lambda}+\varepsilon \bar{h}}-u^{\bar{\lambda}}}{\varepsilon}=\left\{\begin{array}{rlrl}
\frac{x^{2}}{2}-\frac{1}{2} 1-\frac{\sqrt{2}}{2} & \leq x \leq 1, \\
0 & 0 & \leq x \leq 1-\frac{\sqrt{2}}{2} \\
-\left(\frac{x^{2}}{2}+\frac{x}{2}\right) & -1 & \leq x \leq 0 .
\end{array}\right.
$$

By Weiss [1], we know that the Hausdorff dimension of $\Gamma=\partial\{u>0\} \cup \partial\{u<0\}$ is less than or equal to $n-1$ and by Edquist et al. [2] the regularity of the free boundary is $C^{1}$. Let $d \Gamma$ denote the measure $d \Gamma=\mathcal{H}^{n-1}\llcorner\Gamma$; the restriction of the $(n-1)$-dimensional Hausdorff measure $\mathcal{H}^{n-1}$ on the set $\Gamma$. Moreover, let $v_{1}$ be the unit normal exterior to $\partial\{u>0\}$ and $v_{2}$ be the unit normal to $\partial\{u<0\}$ exterior to $\{u<0\}$.

Theorem 3.5. Assume that the free boundary points are one-phase points, and let $\delta$ be the same as defined in Remark 4. Then, we have

$$
\frac{\left.\chi_{\left\{u^{\bar{\lambda}}+\varepsilon \bar{h}\right.}>0\right\}}{\varepsilon}-\chi_{\left\{u^{\bar{\lambda}}>0\right\}} \rightarrow-\frac{1}{\lambda+} \frac{\partial \delta}{\partial v_{1}} d \Gamma^{+},
$$

weakly in $H^{-1}(\Omega)$ as $\varepsilon \rightarrow 0$. In addition

$$
\frac{\chi_{\left\{u^{\bar{\lambda}+\varepsilon \bar{h}}<0\right\}}-\chi_{\left\{u^{\bar{\lambda}}<0\right\}}}{\varepsilon} \rightarrow \frac{1}{\lambda^{-}} \frac{\partial \delta}{\partial v_{2}} d \Gamma^{-} .
$$

Proof. To begin with, observe that

$$
\begin{gathered}
\Delta u^{\bar{\lambda}}=\lambda^{+} \chi_{\left\{u^{\bar{\lambda}}>0\right\}}-\lambda^{-} \chi_{\left\{u^{\bar{\lambda}}<0\right\}^{\prime}} \\
\Delta u^{\bar{\lambda}+\varepsilon \bar{h}}=\left(\lambda^{+}+\varepsilon h_{1}\right) \chi_{\left\{u^{\bar{\lambda}+\varepsilon \bar{h}}>0\right\}}-\left(\lambda^{-}+\varepsilon h_{2}\right) \chi_{\left\{u^{\bar{\lambda}+\varepsilon \bar{h}}<0\right\}} .
\end{gathered}
$$

Then, for a test function $\phi \in H_{0}^{1}(\Omega)$ one obtains

$$
\begin{aligned}
& \int_{\Omega} \Delta\left(\frac{u^{\bar{\lambda}+\varepsilon \bar{h}}-u^{\bar{\lambda}}}{\varepsilon}\right) \phi d x=\int_{\Omega} h_{1} \chi_{\left\{u^{\bar{\lambda}+\varepsilon \bar{h}}>0\right\}} \phi d x-\int_{\Omega} h_{2} \chi_{\left\{u^{\bar{\lambda}+\varepsilon \bar{h}}<0\right\}} \phi d x \\
& +\int_{\Omega} \frac{\lambda^{+}}{\varepsilon}\left(\chi_{\left\{u^{\overline{\bar{x}}+\varepsilon \bar{h}}>0\right\}}-\chi_{\left\{u^{\overline{\bar{x}}}>0\right\}}\right) \phi d x-\int_{\Omega} \frac{\lambda^{-}}{\varepsilon}\left(\chi_{\left\{u^{\bar{\lambda}+\varepsilon \bar{h}}<0\right\}}-\chi_{\left\{u^{\bar{\lambda}}<0\right\}}\right) \phi d x .
\end{aligned}
$$

The left-hand side of Equation 1.27 is

$$
\int_{\Omega} \Delta\left(\frac{u^{\bar{\lambda}+\varepsilon \bar{h}}-u^{\bar{\lambda}}}{\varepsilon}\right) \phi d x=-\int_{\Omega} \nabla\left(\frac{u^{\bar{\lambda}+\varepsilon \bar{h}}-u^{\bar{\lambda}}}{\varepsilon}\right) \nabla \phi d x .
$$


Let $\varepsilon \rightarrow 0$, in (1.27); then, by the notations introduced in Remark 4, one has

$$
\begin{aligned}
& -\int_{\Omega} \nabla \delta \nabla \phi d x=\int_{\Omega} h_{1} \chi_{\left\{u^{\bar{\lambda}}>0\right\}} \phi d x-\int_{\Omega} h_{2} \chi_{\left\{u^{\bar{\lambda}}<0\right\}} \phi d x \\
& +\lim _{\varepsilon \rightarrow 0} \int_{\Omega} \frac{\lambda^{+}}{\varepsilon}\left(\chi_{\left\{u^{\bar{\lambda}+\varepsilon \bar{h}}>0\right\}}-\chi_{\left\{u^{\bar{\lambda}>0}\right\}}\right) \phi d x-\lim _{\varepsilon \rightarrow 0} \int_{\Omega} \frac{\lambda^{-}}{\varepsilon}\left(\chi_{\left\{u^{\bar{\lambda}+\varepsilon \bar{h}}<0\right\}}-\chi_{\left\{u^{\bar{\lambda}}<0\right\}}\right) \phi d x .
\end{aligned}
$$

Integrating by parts gives

$$
\begin{aligned}
-\int_{\Omega} \nabla \delta \nabla \phi d x= & \int_{\left\{u^{\bar{\lambda}}>0\right\}} \Delta \delta \phi d x-\int_{\partial\left\{u^{\bar{\lambda}}>0\right\}} \frac{\partial \delta}{\partial v_{1}} \phi d \sigma \\
& +\int_{\left\{u^{\bar{\lambda}}<0\right\}} \Delta \delta \phi d x-\int_{\partial\left\{u^{\bar{\lambda}}<0\right\}} \frac{\partial \delta}{\partial v_{2}} \phi d \sigma \\
& =\int_{\Omega} h_{1} \chi_{\left\{u^{\bar{\lambda}}>0\right\}} \phi d x-\int_{\Omega} h_{2} \chi_{\left\{u^{\bar{\lambda}}<0\right\}} \varphi d x \\
& +\lim _{\varepsilon \rightarrow 0} \int_{\Omega} \frac{\lambda^{+}}{\varepsilon}\left(\chi_{\left\{u^{\bar{\lambda}+\varepsilon \bar{h}}>0\right\}}-\chi_{\left\{u^{\bar{\lambda}}>0\right\}}\right) \phi d x \\
& -\lim _{\varepsilon \rightarrow 0} \int_{\Omega} \frac{\lambda^{-}}{\varepsilon}\left(\chi_{\left\{u^{\bar{\lambda}+\varepsilon \bar{h}}<0\right\}}-\chi_{\left\{u^{\bar{\lambda}}<0\right\}}\right) \phi d x .
\end{aligned}
$$

In the view of Remark 4, we have

$$
\begin{aligned}
& \lim _{\varepsilon \rightarrow 0}\left[\int_{\Omega} \frac{\lambda^{+}}{\varepsilon}\left(\chi_{\left\{u^{\bar{\lambda}+\bar{h}}>0\right\}}-\chi_{\left\{u^{\bar{\lambda}}>0\right\}}\right) \phi d x-\int_{\Omega} \frac{\lambda^{-}}{\varepsilon}\left(\chi_{\left\{u^{\bar{\lambda}+\varepsilon \bar{h}}<0\right\}}-\chi_{\left\{u^{\bar{\lambda}}<0\right\}}\right) \phi d x\right] \\
& =-\int_{\partial\{u>0\}} \phi \frac{\partial \delta}{\partial v_{1}} d \sigma+\int_{\partial\{u>0\}} \phi \frac{\partial \delta}{\partial v_{2}} d \sigma .
\end{aligned}
$$

Finally, we conclude that

$$
\lim _{\varepsilon \rightarrow 0} \int_{\Omega} \frac{\lambda^{+}}{\varepsilon}\left(\chi_{\left\{u^{\bar{\lambda}+\varepsilon \bar{h}}>0\right\}}-\chi_{\left\{u^{\bar{\lambda}}>0\right\}}\right) \phi d x=-\int_{\partial\{u>0\}} \phi \frac{\partial \delta}{\partial v_{1}} d \sigma,
$$

and

$$
\lim _{\varepsilon \rightarrow 0} \int_{\Omega} \frac{\lambda^{-}}{\varepsilon}\left(\chi_{\left\{u^{\bar{\lambda}+\varepsilon \bar{h}}<0\right\}}-\chi_{\left\{u^{\bar{\lambda}}<0\right\}}\right) \phi d x=\int_{\partial\{u<0\}} \phi \frac{\partial \delta}{\partial v_{2}} d \sigma .
$$

\section{Acknowledgements}

The author thanks Henrik Shahgholian for initiating this work and for useful suggestions. Moreover, the author would like to express his great sense of gratitude to the referees for carefully reading the article and coming with many helpful suggestions.

\section{Competing interests}

The author declares that they have no competing interests.

Received: 11 January 2011 Accepted: 29 June 2011 Published: 29 June 2011

\section{References}

1. Weiss GS: An obstacle-problem-like equation with two phases: pointwise regularity of the solution and an estimate of the Hausdorff dimension of the free boundary. Interfaces Free Bound 2001, 3:121-128.

2. Edquist $\mathrm{A}$, Lindgren $\mathrm{E}$, Shahgholian $\mathrm{H}$ : On the two-phase obstacle problem with coefficents below the lipschitz threshold. Annales de l'Institut Henri Poincare (C) Non Linear Anal 2009, 26:2359-2372.

3. Shahgholian H, Uraltseva N, Weiss GS: Global solutions of an obstacle-problem-like equation with two phases. Monatsh. Math 2004, 142:27-34.

4. Shahgholian H, Weiss GS: The two-phase membrane problem-an intersection-comparison approach to the regularity at branch points. Adv Math 2006, 205:487-503. 
5. Uraltseva N: Two-phase obstacle problem, function theory and phase transitions. J Math Sci 2001, 106:3073-3077.

6. Bozorgnia F: Numerical solution of two-phase membrane problem. Appl Numer Math 2011, 61:92-107.

7. Stojanovic S: Perturbation formula for regular free boundaries in elliptic and parabolic obstacle problems. SIAM J Control Optim 1997, 35:2086-2100.

8. Gilbarg D, Trudinger N: Elliptic Partial Differential Equations of Second Order. Springer, New York; 1983.

9. Blank I: Sharp results for the regularity and stability of the free boundary in the obstacle problem. Indiana Univ Math J 2001, 50(3):1077-1112

10. Evans LC, Gariepy R: Measure Theory and Fine Properties of Functions. CRC Press, Boca Raton; 1992.

doi:10.1186/1687-1847-2011-19

Cite this article as: Bozorgnia: Perturbation formula for the two-phase membrane problem. Advances in Difference Equations 2011 2011:19.

\section{Submit your manuscript to a SpringerOpen ${ }^{\circ}$} journal and benefit from:

- Convenient online submission

- Rigorous peer review

- Immediate publication on acceptance

- Open access: articles freely available online

- High visibility within the field

- Retaining the copyright to your article

Submit your next manuscript at $\gg$ springeropen.com 